with some possible self-interest, such as manufacturers of equipment; and the division of the meeting into two distinct activities, namcly, a survey session, with three contributions, intended to set the current scene, and a series of "case studies"-short contributions in which successful applications of on-line methods in a variety of disciplines were presented.

In a comprehensive review of the present range and facilities of minicomputers-defined as machines possessing input/output channels and "interrupt" (externally initiated program branching) capabilities - D. Turtle (Electrical Research Association) drew attention to the future potentialities of the range of microprocessors which may, before long, provide special purpose systems at a cost of around $£ 1,000$ or less by contrast with the present costs of small computer systems which run, typically, from around $£ 8,000$ for a machine with two registers, an eight-bit word, and sixteen input/ output channels to perhaps $£ 30,000$ for one with sixteen registers, a twentyfour-bit word, and 256 input/output channels.

With the universal use of large-scale integrated circuits and the introduction of new, fast and cheap semiconductor random access and read only memories the possibilities for further reductions in cost and increases in systems capability are by no means exhausted. At one end of the spectrum the system of the future will be characterized by an arrangement in which all component systems (central processor, input/output processors, special arithmetic units, fast and slow stores) will communicate directly with each other, so that many processes may be carried out simultaneously. At the other end, small microprocessors, specifically designed for each application and comprising a few integrated circuits on a single circuit board, will be powerful enough to exert a supervisory and controlling function for many types of experiments.

The problems of physical interconnexion of apparatus and computer, which were considered by J. M. Richards (Computer Analysts and Programmers), offer a choice of two solutions: direct interfacing to the computer's input/output channels for maximum data transfer rates, or use of an intermediate standard interface system. The universality of the latter approach, pioneered by the nuclear physicists and resulting in the internationally standardized CAMAC system, makes it attractive to anybody who can accept the small reduction in the rate of data transfer. One of the benefits of standard interface systems lies in the relative ease with which an experiment can be transferred from one computer to another.

Having successfully linked his apparatus to his computer the experimenter still has to write his program, for he will rarcly be content to use a manufacturer's application package (even if he can afford it). By means of an elaborate flow chart E. G. Murphy (UKAEA) gave guidance about the many choices now open. Only a few years ago use of assembler language was virtually compulsory, but now alternative approaches have become available to the experimenter. The choice of a good operating system and a realistic assessment of the maximum data rates required are all-important in this area.

In the session of case studies C. Derrett (MRC Air Pollution Unit, London) presented an attractively simple approach to the use of an instrument-linked computer system by non-specialists, based on an extension of the well known and simple BASIC programming language to include peripheral handling routines by means of simple CALL statements. At the opposite end of the spectrum of complexity stood two contributions from UKAEA staff at Culham describing a large data acquisition system, and the monitoring and control of a fusion experiment.

The meeting, which attracted more than 200 participants, illustrated clearly that the day when some kind of computing apparatus will form part of almost every experiment is rapidly approaching. In the meantime there seem to be many scientists who, having become convinced on-line experimenters, are willing to let their experience help those who have yet to take the first step.

\title{
More About Cygnus X-3 Radio Flares
}

SINCE the great radio flares of Cygnus $\mathrm{X}-3$ in September and October last year, radio astronomers have kept a watch on the source for more unusual events. These have not as yet materialized. But as a result of the quiescent state of Cyg X-3 since October, Lequeux et al. have been able to carry out absorption measurements at $21 \mathrm{~cm}$ which establish a reliable lower limit on the distance to the source. The same team carried out similar measurements during the flares; but the work described in next Monday's Nature Physical Science (January 29) provides the first proper base line for measurements of this kind, because the observed properties of Cyg X-3 during the flares can hardly be regarded as typical. From these measurements of the absorption between the Earth and Cyg X-3, it is clear that the source is at least $11 \mathrm{kpc}$ away, although it is not clear whether it lies inside or beyond the spiral arm of our Galaxy

\section{PLATE TECTONICS}

\section{Thickening Lithosphere?}

from our Geomagnetism Correspondent WiTH the publication of some new comments and calculations by Forristall (J. Geophys. Res., 77, 6407; 1972), the search for the most plausible plate tectonic mechanism can be said to have taken another small step forward, although, as Forristall himself freely admits, some may feel it to be a step backwards, or at least sideways. This is not because he has returned to some mcchanism now discredited by geophysicists ; on the contrary, he supports the plausibility of the newest idea of all -active, as opposed to passive, lithospheric spreading. The problem is that the plausibility seems to require variability in the thickness of the oceanic lithosphere, an idea which Forristall feels is "certain to be controversial".

According to Forristall's definition, passive spreading is the old mechanism whereby the lithosphere is carried along by convection in the softer asthenosphere below. In this case the velocity in the asthenosphere must increase for some distance below the lithosphereasthenosphere boundary in order to produce the shear stress necessary to move the lithosphere along. One problem with this is that the resulting high aspect ratio cell is unlikely to be stable ; and yet convection extending into the lower mantle, although not entirely discredited, also has its problems. Active spreading, on the other hand, assumes the lithosphere itself to be the prime mover, the driving force being the gravitational forces at elevated ridges or, alternatively, at the descending litho-

which lies at about that distance in the same direction.

Also in next Monday's issue, Bash and Ghigo present observations of the historic flares themselves, at $365 \mathrm{MHz}$. These show the full extent of the second and larger flare, which lasted for more than two weeks (see diagram). More detailed observations have revealed a triple structure within this broad peak, which was also much greater in magnitude than the first event.

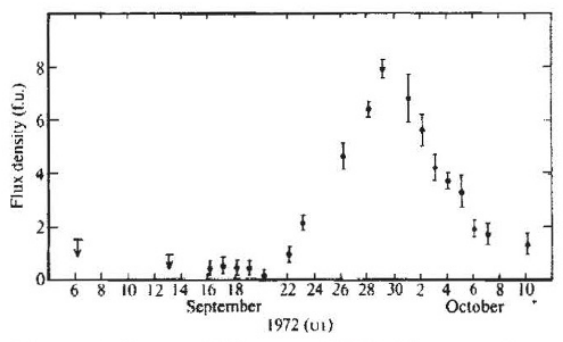

Second flare of Cygnus X-3. Observations at $365 \mathrm{MHz}$. 